Y. Kitaoka

Nagoya Math. J.

Vol. 95 (1984), 73-84

\title{
DIRICHLET SERIES IN THE THEORY OF SIEGEL MODULAR FORMS
}

\section{YOSHIYUKI KITAOKA}

We are concerned with Dirichlet series which appear in the Fourier expansion of the non-analytic Eisenstein series on the Siegel upper half space $H_{m}$ of degree $m$. In the case of $m=2$ Kaufhold [1] evaluated them. Here we treat the general cases by a different method.

For a rational matrix $R$ we denote the product of denominators of elementary divisiors of $R$ by $\nu(R)$. For a half-integral symmetric matrix $T^{(n)}$ we put

$$
b(s, T)=\sum \nu(R)^{-s} e(\sigma(T R)),
$$

where $R$ runs over $n \times n$ rational symmetric matrices modulo 1 and $\sigma$ means the trace, and $e(z)$ is $\exp (2 \pi i z)$. If $\operatorname{Re} s>n+1$, then $b(s, T)$ is absolutely convergent. For a rational symmetric matrix $R$ there is a unique decomposition $R \equiv \sum R_{p} \bmod 1$ where $R_{p}$ is a rational symmetric matrix such that $\nu\left(R_{p}\right)$ is a power of prime $p$. Therefore we have a decomposition

$$
\begin{aligned}
& b(s, T)=\prod b_{p}(s, T), \\
& b_{p}(s, T)=\sum \nu(R)^{-s} e(\sigma(T R)) .
\end{aligned}
$$

where $R$ runs over rational symmetric matrices modulo 1 such that $\nu(R)$ is a power of prime $p$. Our aim is to give $b_{p}(s, T)$ in a form easy to see. Shimura [7] also treats $b_{p}(s, T)$ in a more general situation. $b_{p}(s, T)$ here is a special case $\alpha_{0}$, Case $\mathrm{SP}$ in [7]. His results about $\alpha_{0}$ are weaker than ours.

Generalized confluent hypergeometric functions in the Fourier expansion of the non-analytic Eisenstein series are investigated by Shimura [6].

The author would like to thank Professor G. Shimura who read the

Received April 22, 1983. 
first version of this paper and offered suggestions.

TheOREM 1. Let $T_{1}^{(n-1)}$ be a half-integral symmetric matrix and $T^{(n)}$ $=\left(\begin{array}{ll}T_{1} & 0 \\ 0 & 0\end{array}\right)$. Then we have

$$
b_{p}(s, T)=\left(1-p^{-s}\right)\left(1+p^{1-s}\right)\left(1-p^{n+1-2 s}\right)^{-1} b_{p}\left(s-1, T_{1}\right) .
$$

We prepare some lemmas to prove this theorem. Put $C(k ; p)=$ $\left\{C \in M_{k}(Z)|| C \mid\right.$ is a power of $\left.p\right\}$ and

$$
\Lambda_{k}=\left\{S \in M_{k}(Z){ }^{t} S=S\right\} .
$$

The following lemma is known ([1], [5]).

Lemma 1. $b_{p}(s, T)=\sum|C|^{-s} e\left(\sigma\left(T C^{-1} D\right)\right)$,

where $C, D$ run over $S L_{n}(Z) \backslash C(n ; p),\left\{D \in M_{n}(Z) \mid C^{-1} D={ }^{t}\left(C^{-1} D\right)\right.$ and $(C, D)$ is primitive $\bmod C \Lambda_{n}$ respectively.

$$
\prod_{k=0}^{n-1}\left(1-p^{k-s}\right)^{-1} b_{p}(s, T)=\sum|C|^{-s} e\left(\sigma\left(T C^{-1} D\right)\right),
$$

where $C, D$ run over $S L_{n}(Z) \backslash C(n ; p),\left\{D \in M_{n}(Z) \mid C^{-1} D={ }^{t}\left(C^{-1} D\right)\right\} \bmod C \Lambda_{n}$ respectively.

The next lemma is easy.

LEMMA 2. As representatives of $S L_{n}(Z) \backslash C(n ; p)$ we can choose

$$
C=\left(\begin{array}{ll}
C_{1}^{(n-1)} & 0 \\
C_{3} & C_{4}
\end{array}\right)
$$

where $C_{1}, C_{4}$ and $C_{3}$ run oner $S L_{n-1}(Z) \backslash C(n-1 ; p), C(1 ; p)$ and $M_{1, n-1}(Z)$ $\bmod M_{1, n-1}(Z) C_{1}$ respectively.

Lemma 3. For $C=\left(\begin{array}{ll}C_{1}^{(n-1)} & 0 \\ C_{3} & C_{4}\end{array}\right) \in C(n ; p)$ we can choose as representatives of $\left\{D \in M_{n}(Z) \mid C^{-1} D={ }^{t}\left(C^{-1} D\right)\right\} \bmod C \Lambda_{n}$

$$
D=\left(\begin{array}{ll}
D_{1}^{(n-1)} & D_{2} \\
D_{3} & D_{4}
\end{array}\right)
$$

where $D_{1}, D_{2}$ and $D_{4}$ run over $\left\{D_{1} \in M_{n-1}(Z) \mid C_{1}^{-1} D_{1}={ }^{t}\left(C_{1}^{-1} D_{1}\right)\right\} \bmod C_{1} A_{n-1}$, $\left\{D_{2} \in M_{n-1,1}(Z) \mid C_{4}{ }^{t} D_{2}+C_{3}{ }^{t} D_{1} \in M_{1, n-1}(Z)^{t} C_{1}\right\} \bmod C_{1} M_{n-1,1}(Z)$ and $Z \bmod C_{4}$ respectively and then $D_{3}=\left(C_{4}{ }^{t} D_{2}+C_{3}{ }^{t} D_{1}\right)^{t} C_{1}^{-1}$. 
Proof. Since $C^{-1}=\left(\begin{array}{cc}C_{1}^{-1} & 0 \\ -C_{4}^{-1} C_{3} C_{1}^{-1} & C_{4}^{-1}\end{array}\right)$, we have

$$
C^{-1} D=\left(\begin{array}{cc}
C_{1}^{-1} D_{1} & C_{1}^{-1} D_{2} \\
-C_{4}^{-1}\left(C_{3} C_{1}^{-1} D_{1}-D_{3}\right) & -C_{4}^{-1}\left(C_{3} C_{1}^{-1} D_{2}-D_{4}\right)
\end{array}\right) .
$$

Since $C^{-1} D$ is symmetric, $C_{1}^{-1} D_{1}$ is symmetric and $D_{3}=\left(C_{4}^{t} D_{2}+C_{3}{ }^{t} D_{1}\right)^{t} C_{1}^{-1}$. For an integral symmetric matrix $S=\left(\begin{array}{cc}S_{1}^{(n-1)} & S_{2} \\ { }^{t} S_{2} & S_{4}\end{array}\right)$,

$$
C S=\left(\begin{array}{cc}
C_{1} S_{1} & C_{1} S_{2} \\
C_{3} S_{1}+C_{4}{ }^{t} S_{2} & C_{3} S_{2}+C_{4} S_{4}
\end{array}\right) \text { holds . }
$$

From these follows easily our lemma.

The next lemma is an immediate corollary.

Lemma 4. Let $C_{1} \in C(n-1 ; p), D_{1} \in M_{n-1}(Z)$ and $C_{4} \in C(1 ; p)$. Denote by $x\left(C_{1}, D_{1}, C_{4}\right)$ the number of elements of the set

$$
\begin{gathered}
\left\{D_{2} \in M_{n-1,1}(Z) \bmod C_{1} M_{n-1,1}(Z), C_{3} \in M_{1, n-1}(Z) \bmod M_{1, n-1}(Z) C_{1}\right. \\
\text { such that } \left.C_{4}{ }^{t} D_{2}+C_{3}{ }^{t} D_{1} \in M_{1, n-1}(Z)^{t} C_{1}\right\}
\end{gathered}
$$

Then the number of $C=\left(\begin{array}{ll}C_{1} & 0 \\ C_{3} & C_{4}\end{array}\right), D=\left(\begin{array}{ll}D_{1} & D_{2} \\ D_{3} & D_{4}\end{array}\right)$ where $C_{3}, D$ run over $M_{1, n-1}(Z) \bmod M_{1, n-1}(Z) C_{1},\left\{D \in M_{n}(Z) \bmod C A_{n} \mid C^{-1} D={ }^{t}\left(C^{-1} D\right)\right\}$ respectively is $C_{4} x\left(C_{1}, D_{1}, C_{4}\right)$.

Lemma 5. Let $R$ be a rational symmetric matrix and $C_{i}^{-1} D_{i}=R$ for $C_{i}, D_{i} \in M_{n}(Z)(i=1,2)$. If $\left(C_{1}, D_{1}\right)$ is primitive then $\left(C_{2}, D_{2}\right)=W\left(C_{1}, D_{1}\right)$ for some $W \in M_{n}(Z)$.

Proof. This is well known [5]).

Lemma 6. Let $W \in C(n-1 ; p), C_{4} \in C(1 ; p), C_{1} \in C(n-1 ; p)$ and $D_{1} \in$ $M_{n-1}(Z)$ such that $C_{1}^{-1} D_{1}$ is symmetric and $\left(C_{1}, D_{1}\right)$ is primitive. Then we have

$$
x\left(W C_{1}, W D_{1}, C_{4}\right)=\left|W C_{1}\right| \prod_{i=1}^{n-1}\left(C_{4}, w_{i}\right)
$$

where $\left\{w_{i}\right\}$ is the set of elementary divisors of $W$.

Proof. Let $A_{1}, B_{1} \in M_{n-1}(Z)$ such that $\left(\begin{array}{ll}A_{1} & B_{1} \\ C_{1} & D_{1}\end{array}\right) \in S p_{n-1}(Z)$. Suppose $z^{t} D_{1}=w^{t} C_{1}$ for $z, w \in M_{1, n-1}(Z)$; then $z=z\left({ }^{t} D_{1} A_{1}-{ }^{t} B_{1} C_{1}\right)=w^{t} C_{1} A_{1}$ 
$z^{t} B_{1} C_{1}=\left(w^{t} A_{1}-z^{t} B_{1}\right) C_{1} \in M_{1, n-1}(Z) C_{1}$. Conversely, suppose $z=x C_{1}$ for $z, x \in M_{1, n-1}(Z)$; then $z^{t} D_{1}=x C_{1}{ }^{t} D_{1}=x D_{1}{ }^{t} C_{1} \in M_{1, n-1}(Z)^{t} C_{1}$. Thus we have proved that for $z \in M_{1, n-1}(Z)$

$$
z^{t} D_{1} \in M_{1, n-1}(Z)^{t} C_{1} \quad \text { iff } z \in M_{1, n-1}(Z) C_{1} .
$$

Next we show that for $D_{2} \in M_{n-1,1}(Z)$ there exists $C_{3} \in M_{1, n-1}(Z)$ such that $C_{4}{ }^{t} D_{2}+C_{3}{ }^{t}\left(W D_{1}\right) \in M_{1, n-1}(Z)^{t}\left(W C_{1}\right)$ iff $C_{4}{ }^{t} D_{2}{ }^{t} W^{-1} \in M_{1, n-1}(Z)$. The "only if" part is trivial. Suppose $C_{4}^{t} D_{2}^{t} W^{-1}=y \in M_{1, n-1}(Z)$; then $y-y A_{1}^{t} D_{1}=$ $-y B_{1}{ }^{t} C_{1}$ implies $C_{4}{ }^{t} D_{2}+(-y A)^{t}\left(W D_{1}\right)=-y B_{1}{ }^{t}\left(W C_{1}\right) \in M_{1, n-1}(Z)^{t}\left(W C_{1}\right)$. Hence we can take $-y A_{1}$ as $C_{3}$.

Lastly suppose that $D_{2} \in M_{n-1,1}(Z), C_{3, i} \in M_{1, n-1}(Z)$ satisfy

$$
C_{4}{ }^{t} D_{2}+C_{3, i}{ }^{t}\left(W D_{1}\right) \in M_{1, n-1}(Z)^{t}\left(W C_{1}\right) \quad(i=1,2),
$$

then $\left(C_{3,1}-C_{3,2}\right)^{t} D_{1} \in M_{1, n-1}(Z)^{t} C_{1}$ and then $C_{3,1}-C_{3,2} \in M_{1, n-1}(Z) C_{1}$. Therefore

$$
\begin{aligned}
& x\left(W C_{1}, W D_{1}, C_{4}\right) \\
& \quad=|W| \sharp\left\{D_{2} \in M_{n-1,1}(Z) \bmod W C_{1} M_{n-1,1}(Z) \mid C_{4}{ }^{t} D_{2}{ }^{t} W^{-1} \in M_{1, n-1}(Z)\right\} .
\end{aligned}
$$

Let $W=U W_{0} V$ where

$$
U, V \in G L_{n}(Z), \quad W_{0}=\left[\begin{array}{lll}
w_{1} & & \\
\ddots & \\
& & \\
& w_{n-1}
\end{array}\right] \text { and } \operatorname{put}{ }^{t} D_{2}{ }^{t} U^{-1}=\left(y_{1}, \cdots, y_{n-1}\right) .
$$

${ }^{t} D_{2}{ }^{t} W^{-1}=\left(\cdots, y_{i} / w_{i}, \cdots\right){ }^{t} V^{-1}$ implies

$$
\begin{aligned}
& x\left(W C_{1}, W D_{1}, C_{4}\right) \\
& =|W| \#\left\{\left(y_{1}, \cdots, y_{n-1}\right) \in M_{1, n-1}(Z) \bmod M_{1, n-1}(Z)^{t} C_{1}^{t} V W_{0} \mid C_{4} y_{i} \equiv 0 \bmod w_{i}\right\} \\
& =|W|\left[M_{1, n-1}(Z): M_{1, n-1}(Z)^{t} C_{1}{ }^{t} V W_{0}\right] \mid \\
& \quad\left[M_{1, n-1}(Z):\left\{\left(y_{1}, \cdots, y_{n-1}\right) \in M_{1, n-1}(Z) \mid y_{i} \equiv 0 \bmod ^{*} w_{i} /\left(C_{4}, w_{i}\right)\right\}\right] \\
& =\left|C_{1} W\right| \prod\left(C_{4}, w_{i}\right) .
\end{aligned}
$$

Proof of Theorem 1. From above lemmas follows that

$$
\begin{aligned}
& \prod_{k=0}^{n-1}\left(1-p^{k-s}\right)^{-1} b_{p}(s, T) \\
& \quad=\sum\left|C_{1}\right|^{-s} C_{4}^{1-s} e\left(\sigma\left(T_{1} C_{1}^{-1} D_{1}\right)\right) x\left(C_{1}, D_{1}, C_{4}\right),
\end{aligned}
$$

where $C_{1}, D_{1}, C_{4}$ run over $S L_{n-1}(Z) \backslash C(n-1 ; p),\left\{D_{1} \in M_{n-1}(Z) \bmod C_{1} \Lambda_{n-1} \mid\right.$ $\left.C_{1}^{-1} D_{1}={ }^{t}\left(C_{1}^{-1} D_{1}\right)\right\}$ and $C(1 ; p)$ respectively

$$
=\sum\left|W C_{1}\right|^{-s} C_{4}^{1-s} e\left(\sigma\left(T_{1} C_{1}^{-1} D_{1}\right)\right) x\left(W C_{1}, W D_{1}, C_{4}\right),
$$


where $C_{1}, D_{1}, C_{4}$ run over the same set as above with an additional condition that $\left(C_{1}, D_{1}\right)$ is primitive, and $W$ runs over $S L_{n-1}(Z) \backslash C(n-1 ; p)$

$$
=\sum\left|C_{1}\right|^{1-s} e\left(\sigma\left(T_{1} C_{1}^{-1} D_{1}\right)\right) \cdot \sum|W|^{1-s} C_{4}^{1-s} \prod\left(C_{4}, w_{i}\right),
$$

where $C_{1}, D_{1}, C_{4}, W$ run over the above set and $\left\{w_{i}\right\}$ is the set of elementary divisors of $W$.

Thus we have proved that $b_{p}(s, T) b_{p}\left(s-1, T_{1}\right)^{-1}$ is independent of $T_{1}$. Hence by the formula of $b_{p}(s, 0)$ ([7]) or evaluating $b_{p}(s, T), b_{p}\left(s, T_{1}\right)$ for

$$
T_{1}=\frac{1}{2}\left(\begin{array}{lllll}
0 & 1 & & & \\
1 & 0 & & & \\
& & \ddots & & \\
& & & 0 & 1 \\
& & & 1 & 0
\end{array}\right) \quad \text { or } \quad \frac{1}{2}\left(\begin{array}{ccccc}
0 & 1 & & & \\
1 & 0 & & & \\
& & \ddots & & \\
& & & 0 & 1 \\
& & & 1 & 0 \\
& & & &
\end{array}\right)
$$

similarly to the proof of the next theorem we have $b_{p}(s, T) b_{p}\left(s-1, T_{1}\right)^{-1}$ $=\left(1-p^{-s}\right)\left(1+p^{1-s}\right)\left(1-p^{n+1-2 s}\right)^{-1}$.

CoRollary 1. Let $T^{(n)}=\left(\begin{array}{ll}T_{1}^{(n-r)} & 0 \\ 0 & 0\end{array}\right)(1 \leqq r<n)$ be a half-integral symmetric matrix. Then we have

$$
\begin{aligned}
b_{p}(s, T)= & \left(1-p^{-s}\right)\left(1+p^{r-s}\right) \prod_{0<i \leqq \min (r-1,[n / 2])}\left(1-p^{2 i-2 s}\right) \\
& \times \prod_{\max (r,[n / 2]+1) \leqq j \leqq[(n+r) / 2]}\left(1-p^{2 j-2 s}\right)^{-1} \\
& \times \prod_{\substack{n+1 \leq k \leq n+r \\
2 \nmid k \in}}\left(1-p^{k-2 s}\right)^{-1} b_{p}\left(s-r, T_{1}\right),
\end{aligned}
$$

where [ ] means the Gauss' symbol.

Proof. By induction it is easy to see

$$
\begin{aligned}
b_{p}(s, T)= & \left(1-p^{-s}\right)\left(1+p^{r-s}\right) \prod_{0<i<r}\left(1-p^{2 i-2 s}\right) \\
& \cdot \prod_{n+1 \leqq j \leqq n+r}\left(1-p^{j-2 s}\right)^{-1} b_{p}\left(s-r, T_{1}\right) .
\end{aligned}
$$

From this follows our formula.

Corollary 2. Let $O^{(n)}$ be the $n \times n$ zero matrix. Then we have

$$
\begin{aligned}
b_{p}\left(s, O^{(n)}\right)= & \left(1-p^{-s}\right) \prod_{\substack{0<k \leqq[n / 2]\\
}}\left(1-p^{2 k-2 s}\right) \\
& \cdot\left\{\left(1-p^{n-s}\right) \prod_{\substack{n+1 \leq j<2 n \\
2 \nmid j}}\left(1-p^{j-2 s}\right)\right\}^{-1} .
\end{aligned}
$$


Proof. $\quad b_{p}\left(s, O^{(1)}\right)=\left(1-p^{-s}\right)\left(1-p^{1-s}\right)^{-1}$ is easy to see. Applying Corollary 1 to $r=n-1, T=O^{(n)}$ we get

$$
b_{p}\left(s, O^{(n)}\right)=\left(1-p^{-s}\right)\left(1-p^{n-s}\right)^{-1} \prod_{1 \leqq k \leqq n-1}\left\{\left(1-p^{2 k-2 s}\right)\left(1-p^{n+k-2 s}\right)^{-1}\right\} .
$$

From this follows our formula.

Remark. In Corollary 1 there is a cancellation

$$
\left(1+p^{r-s}\right)\left(1-p^{2 j-2 s}\right)^{-1}=\left(1-p^{r-s}\right)^{-1} \quad(j=r) \quad \text { if } r \geqq[n / 2]+1 .
$$

In the rest of this paper we will show that $b_{p}(s, T)$ is a polynomial in $p^{-s}$ for regular half-integral symmetric matrices $T$.

Put $E_{n}=\left\{S=\left(s_{i j}\right) \in M_{n}\left(Z_{p}\right) \mid S={ }^{t} S, s_{i i} \in 2 Z_{p}(1 \leqq i \leqq n)\right\}$ and

$$
H_{s}=\left(\begin{array}{lllll}
0 & 1 & & & \\
1 & 0 & & & \\
& & \ddots & & \\
& & & 0 & 1 \\
& & & 1 & 0
\end{array}\right) \in E_{2 s}
$$

For $N \in E_{n}$ we put

$$
\begin{aligned}
\alpha\left(N, H_{s} ; p^{t}\right) & =\left\{T \in M_{2 s, n}\left(Z_{p} /\left(p^{t}\right)\right) \mid H_{s}[T]-N \in p^{t} E_{n}\right\}, \\
B\left(N, H_{s} ; p^{t}\right) & =\left\{T \in \alpha\left(N, H_{s} ; p^{t}\right) \mid T: \text { primitive }\right\} .
\end{aligned}
$$

LEMMA 7. Let $N \in E_{n}$ with $|N| \neq 0$ and $G \in G L_{n}\left(\boldsymbol{Q}_{p}\right) \cap M_{n}\left(Z_{p}\right)$. If $t>$ $\operatorname{ord}_{p}|N|$, we have

$$
\begin{gathered}
\left(p^{t}\right)^{n(n+1) / 2-2 s n} \sharp\left\{T \in \alpha\left(N, H_{s} ; p^{t}\right) \mid M_{2 s, n}\left(Z_{p}\right) \ni T G^{-1}: \text { primitive }\right\} \\
=\left(p^{\operatorname{ord}_{p}|G|}\right)^{n-2 s+1} p^{n(n+1) / 2-2 s n} \sharp B\left(N\left[G^{-1}\right], H_{s} ; p\right) .
\end{gathered}
$$

Proof. Let $T \in M_{2 s, n}\left(Z_{p}\right)$ and suppose that $H_{s}[T]-N \in p^{t} E_{n}$ and $T_{1}=$ $T G^{-1}$ is primitive. Then $H_{s}[T]=N+p^{t} C$ holds for some $C \in E_{n}$ and $H_{s}[T]$ $\equiv N \bmod p^{t}$. Hence $\left|H_{s}\left[T_{1}\right]\right||G|^{2} \equiv|N| \bmod p^{t}$ holds and $2 \operatorname{ord}_{p}|G| \leqq \operatorname{ord}_{p}|N|$ $<t$ follows from $\operatorname{ord}_{p}|N|<t$. Denote by $C_{1}, \cdots, C_{a}$ the representatives of the set $\left\{p^{t} \bar{C}\left[G^{-1}\right] \mid \bar{C} \in E_{n}\right\} \bmod p^{t} E_{n}$, then we have $H_{s}\left[T_{1}\right]=N\left[G^{-1}\right]+$ $p^{t} C\left[G^{-1}\right] \equiv N\left[G^{-1}\right]+C_{k} \bmod p^{t} E_{n}$. Conversely suppose that $T_{1} \in M_{2 s, n}\left(Z_{p}\right)$ and $T_{1}$ is primitive and $H_{s}\left[T_{1}\right] \equiv N\left[G^{-1}\right]+C_{k} \bmod p^{t} E_{n}$, then we have $H_{s}\left[T_{1} G\right] \equiv N \bmod p^{t} E_{n}$. Therefore we get

$$
\begin{aligned}
\sharp\{T \in & \left.M_{2 s, n}\left(Z_{p}\right) \bmod p^{t} M_{2 s, n}\left(Z_{p}\right) G \mid H_{s}[T]-N \in p^{t} E_{n}, T G^{-1} \text { : primitive }\right\} \\
& =\sum_{k=1}^{a} \sharp B\left(N\left[G^{-1}\right]+C_{k}, H_{s} ; p^{t}\right) .
\end{aligned}
$$


Since $C_{k}$ is in $p E_{n}$, by virtue of 2.2 in [2] we have

$$
\begin{gathered}
\left(p^{t}\right)^{n(n+1) / 2-2 s n} \sharp B\left(N\left[G^{-1}\right]+C_{k}, H_{s} ; p^{t}\right) \\
\quad=p^{n(n+1) / 2-2 s n} \sharp B\left(N\left[G^{-1}\right]+C_{k}, H_{s} ; p\right) \\
=p^{n(n+1) / 2-2 s n} \sharp B\left(N\left[G^{-1}\right], H_{s} ; p\right) .
\end{gathered}
$$

Let $p^{a_{1}}, \cdots, p^{a_{n}}$ be elementary divisors of $G$, then from the definition of $a$ follows immediately

$$
\begin{aligned}
a & =\#\left[\left\{p^{t}\left(c_{i j} p^{-a_{i}-a_{j}}\right) \mid\left(c_{i j}\right) \in E_{n}\right\} \bmod p^{t} E_{n}\right] \\
& =\left(p^{\text {ord } p|G|}\right)^{n+1} .
\end{aligned}
$$

Thus we have

$$
\begin{aligned}
& \left(p^{\text {ord } p|G|}\right)^{2 s} \sharp\left\{T \in \alpha\left(N, H_{s} ; p^{t}\right) \mid M_{2 s, n}\left(Z_{p}\right) \ni T G^{-1}: \text { primitive }\right\} \\
& \quad=\left(p^{\text {ord } p|G|}\right)^{n+1}\left(p^{-t}\right)^{n(n+1) / 2-2 s n} p^{n(n+1) / 2-2 s n} \sharp B\left(N\left[G^{-1}\right], H_{s} ; p\right) .
\end{aligned}
$$

As a corollary we get

Lemma 8. Let $N \in E_{n}$ with $|N| \neq 0$ and $t>\operatorname{ord}_{p}|N|$. Then we have

$$
\begin{aligned}
& \left(p^{t}\right)^{n(n+1) / 2-2 s n} \sharp \alpha\left(N, H_{s} ; p^{t}\right) \\
& \quad=\sum\left(p^{\text {ord }_{p}|G|}\right)^{n+1-2 s} p^{n(n+1) / 2-2 s n} \sharp B\left(N\left[G^{-1}\right], H_{s} ; p\right)
\end{aligned}
$$

where $G$ runs over $G L_{n}\left(Z_{p}\right) \backslash\left\{G L_{n}\left(\boldsymbol{Q}_{p}\right) \cap M_{n}\left(Z_{p}\right)\right\}$.

Proof. Let $T \in \alpha\left(N, H_{s} ; p^{t}\right)$ and suppose that $T G^{-1}$ is primitive for $G \in G L_{n}\left(\boldsymbol{Q}_{p}\right) \cap M_{n}\left(Z_{p}\right)$. For any matrix $T_{1} \equiv T \bmod p^{t} T_{1} G^{-1}$ is also primitive since $2 \operatorname{ord}_{p}|G|<t$ as in the proof of the previous lemma. If $T G_{1}^{-1}$, $T G_{2}^{-1}$ are primitive for $G_{i} \in G L_{n}\left(\boldsymbol{Q}_{p}\right) \cap M_{n}\left(Z_{p}\right)$, then $G_{1} G_{2}^{-1} \in G L_{n}\left(Z_{p}\right)$ since $T G_{1}^{-1}\left(G_{1} G_{2}^{-1}\right)=T G_{2}^{-1}$. Now Lemma 7 completes the proof of Lemma 8 .

Let $H=Z /(p)[e, f]$ be a quadratic space over $Z /(p)$ such that $q(e)=$ $q(f)=0, b(e, f)=1(q(x+y)-q(x)-q(y)=b(x, y))$, and $\bar{H}_{s}=\perp_{s} H$. For a quadratic space $N$ over $Z /(p)$ we put

$$
B\left(N, \bar{H}_{s}\right)=\sharp\left\{\text { isometries form } N \text { to } \bar{H}_{s}\right\} \text {. }
$$

If $N \in E_{n}$, then

$$
q\left(x_{1}, \cdots, x_{n}\right)=\frac{1}{2} N\left[\begin{array}{c}
x_{1} \\
\vdots \\
x_{n}
\end{array}\right]
$$

makes a quadratic space $N^{\prime}$ over $Z /(p)$ corresponding to $N$ and $\sharp B\left(N, H_{s} ; p\right)$ $=B\left(N^{\prime}, \bar{H}_{s}\right)$ holds. 
Lemma 9. Let $N$ be a quadratic space over $Z /(p)$ and $\operatorname{dim} N=n$. Let $N=N_{1} \perp N_{2}$ where $N_{2}$ is a maximal totally singular subspace, that is, $N_{2}$ has a maximal dimension among the subspaces in $N$ such that $q\left(N_{2}\right)=0$. Put $\operatorname{dim} N_{1}=d$ and $\varepsilon=1$ if $N_{1}$ is isometric to $\bar{H}_{k}$ for some $k$ or $d=0$, otherwise $\varepsilon=-1$. Then for a sufficiently large $s$ we have

$$
\begin{aligned}
p^{n(n+1) / 2-2 s n} B\left(N, \bar{H}_{s}\right) & \\
= & \begin{cases}\left(1-p^{-s}\right)\left(1+\varepsilon p^{n-d / 2-s}\right) \prod_{1 \leqq i \leqq n-d / 2-1}\left(1-p^{2 i-2 s}\right) & 2 \mid d, \\
\left(1-p^{-s}\right) \prod_{1 \leqq i \leqq n-(d+1) / 2}\left(1-p^{2 i-2 s}\right) & 2 \nmid d .\end{cases}
\end{aligned}
$$

Proof. Let $p$ be an odd prime. This follows from the proof of Lemma 1 in [2]. For a sufficiently large $s$ there is an isometry $u$ from $N$ into $\bar{H}_{s}$. Let $M$ be the orthogonal complement of $u\left(N_{1}\right)$ in $\bar{H}_{s}$. By the theorem of Witt the isometry class of $M$ is independent of the choice of $u$. Then we have

$$
B\left(N, \bar{H}_{s}\right)=B\left(N_{1}, \bar{H}_{s}\right) B\left(N_{2}, M\right),
$$

where $B\left(N_{2}, M\right)$ is the number of isometries from $N_{2}$ into $M$. Then it is known ([8], [2]).

$$
\begin{aligned}
& p^{d(d+1) / 2-2 s d} B\left(N_{1}, \bar{H}_{s}\right) \\
& = \begin{cases}\left(1-p^{-s}\right)\left(1+\varepsilon p^{d / 2-s}\right) \prod_{1 \leqq k \leqq d / 2-1}\left(1-p^{2 k-2 s}\right) & 2 \mid d>0, \\
\left(1-p^{-s}\right) \prod_{1 \leqq k \leqq(d-1) / 2}\left(1-p^{2 k-2 s}\right) & 2 \nmid d,\end{cases} \\
& p^{-(2 s-d)(n-d)+(n-d)(n-d+1) / 2} B\left(N_{2}, M\right) \\
& = \begin{cases}\prod_{0 \leqq k \leqq n-d-1}\left\{\left(1-\varepsilon p^{n-s-d / 2-k-1}\right)\left(1+\varepsilon p^{n-s-d / 2-k}\right)\right\} & 2 \mid d, \\
\prod_{0 \leqq k \leqq n-d-1}\left(1-p^{2 n-2 s-d-1-2 k}\right) & 2 \nmid d .\end{cases}
\end{aligned}
$$

From this follows our formula. Similarly we get the same formulas for $p=2$. There is nothing to change in the above proof for an odd prime $p$.

Let $T$ be a half-integral symmetric matrix with $|T| \neq 0$. Put

$$
b_{p}\left(s, T ; p^{t}\right)=\sum_{R \bmod p^{t}} \nu\left(p^{-t} R\right)^{-s} e\left(\sigma\left(T\left(p^{-t} R\right)\right)\right),
$$

where $R$ runs over integral symmetric matrices $\bmod p^{t}$. Then it is known ([4]) that for a natural number $s$

$$
\begin{aligned}
b_{p}\left(s, T ; p^{t}\right) & =\left(p^{t}\right)^{n(n+1) / 2-2 n s} \sharp\left\{K^{(n, 2 s)} \bmod p^{t} \mid p^{-t}\left(\frac{1}{2} H_{s}\left[{ }^{t} K\right]+T\right) \in 2^{-1} E_{n}\right\} \\
& =\left(p^{t}\right)^{n(n+1) / 2-2 n s} \sharp \alpha\left(-2 T, H^{s} ; p^{t}\right) .
\end{aligned}
$$


By definition $b_{p}\left(s, T ; p^{t}\right)$ is a polynomial in $p^{-s}$. On the other hand by virtue of Lemma 8,9 there exists a polynomial $f(x, T)$ which depends only on $T$ such that $b_{p}\left(s, T ; p^{t}\right)=f\left(p^{-s}, T\right)$ if $s, t$ are sufficiently large integers. Hence we have $b_{p}\left(s, T ; p^{t}\right)=f\left(p^{-s}, T\right)$ for any $s \in C$, and $b_{p}(s, T)$ $=f\left(p^{-s}, T\right)$ as $t \rightarrow \infty$.

Thus we have proved

TheOREm 2. Let $T^{(n)}$ be a half-integral symmetric matrix with $|T| \neq 0$. Then we have

$$
b_{p}(s, T)=\sum_{G}\left(p^{\mathrm{ord}_{p}|G|}\right)^{n+1-2 s} a\left(-T\left[G^{-1}\right], s\right),
$$

where $G$ runs over $G L_{n}\left(\boldsymbol{Z}_{p}\right) \backslash\left\{G L_{n}\left(\boldsymbol{Q}_{p}\right) \cap M_{n}\left(\boldsymbol{Z}_{p}\right)\right\}$ and $a(T, s)$ is defined as follows. If $T$ is not half-integral, $a(T, s)=0$. If $T$ is half-integral, we define a quadratic space $N$ over $Z /(p)$ with $\operatorname{dim} N=n$ by

$$
q\left(x_{1}, \cdots, x_{n}\right)=T\left[\begin{array}{c}
x_{1} \\
\vdots \\
x_{n}
\end{array}\right] \bmod p, \text { and } N=N_{1} \perp N_{2}
$$

where $N_{2}$ is a maximal totally singular subspace. Put $d=\operatorname{dim} N_{1}$ and $\varepsilon=1$ if $N_{1}$ is a hyperboilc space or $d=0$, otherwise $\varepsilon=-1$. Then we set

$$
a(T, s)= \begin{cases}\left(1-p^{-s}\right)\left(1+\varepsilon p^{n-d / 2-s}\right) \prod_{1 \leqq \imath \geqq n-d / 2-1}\left(1-p^{2 i-2 s}\right) & 2 \mid d, \\ \left(1-p^{-s}\right) \prod_{1 \leqq i \leqq n-(d+1) / 2}\left(1-p^{2 i-2 s}\right) & 2 \nmid d .\end{cases}
$$

In the above formula for $b_{p}(s, T) G$ runs over a finite set.

Corollary. (i) Let $O^{(n)}$ be the $n \times n$ zero matrix. Then

$$
b_{p}\left(s, O^{(n)}\right)=\left(1-p^{-s}\right) \prod_{0<k \leq[n / 2]}\left(1-p^{2 k-2 s}\right)\left\{\left(1-p^{n-s}\right) \prod_{n+1 \frac{1}{2} j<j<2}\left(1-p^{j-2 s}\right)\right\}^{-1} .
$$

Let $T^{(n)}=\left(\begin{array}{ll}T_{1}^{(n-r)} & 0 \\ 0 & 0\end{array}\right)$ be a half-integral symmetric matrix with $\left|T_{1}\right| \neq 0$ $\left(0^{*} \leqq r<n\right)$.

(ii) If $p$ does not divide $\left|2 T_{1}\right|$, then

$$
\begin{aligned}
b_{p}(s, T)= & \left(1-p^{-s}\right) \prod_{1 \leqq j \leq[n / 2]}\left(1-p^{2 j-2 s}\right) \prod_{\substack{n+1 \leq k \leq n+r \\
2 \nmid k}}\left(1-p^{k-2 s}\right)^{-1} \\
& \times \begin{cases}\left(1-\varepsilon\left(T_{1}\right) p^{(n+r) / 2-s}\right)^{-1} & 2 \mid n-r \\
1 & 2 \nmid n-r\end{cases}
\end{aligned}
$$


where $\varepsilon\left(T_{1}\right)=1$ if $T_{1}$ corresponds to a hyperbolic space over $Z /(p)$, and $\varepsilon\left(T_{1}\right)=-1$ otherwise, i.e., $\varepsilon\left(T_{1}\right)=\left(\left((-1)^{(n-r) / 2}\left|2 T_{1}\right|\right) / p\right)$ (Kronecker symbol).

(iii) If $n-r$ is odd, then

$$
\begin{aligned}
b_{p}(s, T)= & \left(\text { polynomial in } p^{-s}\right)\left(1-p^{-s}\right) \prod_{1 \leqq j \leqq[n / 2]}\left(1-p^{2 j-2 s}\right) \\
& \times \prod_{n+1 \leq k \leq n+r}\left(1-p^{k-2 s}\right)^{-1} .
\end{aligned}
$$

(iv) If $n-r$ is even, then

$$
\begin{aligned}
& b_{p}(s, T)=\left(\text { polynomial in } p^{-s}\right) \times\left(1-\eta p^{(n+r) / 2-s}\right)^{-1}\left(1-p^{-s}\right) \\
& \times \prod_{1 \leqq j \leqq n / 2]}\left(1-p^{2 j-2 s}\right) \prod_{\substack{n+1 \leq k \leq s+r \\
2 \nmid k}}\left(1-p^{k-2 s}\right)^{-1},
\end{aligned}
$$

where $\eta$ is defined as follows:

If there is an integral matrix $G^{(n-r)}$ such that $T_{1}\left[G^{-1}\right]$ is half-integral and $\left|2 T_{1}\left[G^{-1}\right]\right|$ is not divided by $p$, then

$$
\eta=\varepsilon\left(T_{1}\left[G^{-1}\right]\right) \quad \text { (in (ii)) . }
$$

( $\eta$ is uniquely determined by $T_{1}$ ).

$$
\text { Otherwise } \eta=0 \text {. }
$$

Especially $\eta=0$ if $\operatorname{ord}_{p}\left|2 T_{1}\right|$ is odd.

Proof. (i) is already proved. (ii) follows from Corollary 1 and Theorem 2. Let $T_{2}^{(n-r)}$ be a half-integral matrix with $\left|T_{2}\right| \neq 0$. If $n-r$ is odd or $p|| 2 T_{2} \mid$, then $a\left(T_{2}, s\right)$ is divided by

$$
\left(1-p^{-s}\right) \prod_{1 \leqq i \leq\lfloor(n-r) / 2]}\left(1-p^{2 i-2 s}\right) \text {. }
$$

(iii) and (iv) for $\eta=0$ follow from this and Corollary 1 and Theorem 2. Suppose that there is an integral matrix $G^{(n-r)}$ such that $T_{1}\left[G^{-1}\right]$ is halfintegral and $\left|2 T_{1}\left[G^{-1}\right]\right|$ is not divided by $p$. Then

$$
a\left(T_{1}\left[G^{-1}\right], s\right)=\left(1-p^{-s}\right)\left(1+\varepsilon\left(T_{1}\left[G^{-1}\right]\right) p^{(n-r) / 2-s}\right) \prod_{1 \leqq i \leqq(n-r) / 2-1}\left(1-p^{2 i-2 s}\right) .
$$

The coset $G_{n-r}\left(Z_{p}\right) G$ is not necessarily unique, but $\varepsilon\left(T_{1}\left[G^{-1}\right]\right)$ depends only on $T_{1}$. Taking these terms into account, we complete the proof of the case $\eta \neq 0$.

Remark 1. Let $n=2 k$ be an even integer and $T^{(n)}$ a half-integral symmetric regular matrix. Let $L=Z_{p}\left[e_{1}, \cdots, e_{n}\right]$ be a free module over 
and define a bilinear form $B\left(e_{i}, e_{j}\right)$ on it by $\left(B\left(e_{i}, e_{j}\right)\right)=2 T$. Then there an integral matrix $G$ such that $T\left[G^{-1}\right]$ is half-integral and $p \nmid\left|2 T\left[G^{-1}\right]\right|$ and only if there is a unimodular lattice $M$ such that $M \supset L$ and the ?m of $M$ is $2 Z_{p}$. A corresponding matrix to $M$ is diag $[1, \cdots, 1, \delta]$ $\left.\vdots Z_{p}^{\times}\right)$if $p \neq 2$,

$$
\left\{\begin{array}{l}
\operatorname{diag}\left[\left(\begin{array}{ll}
0 & 1 \\
1 & 0
\end{array}\right), \cdots,\left(\begin{array}{ll}
0 & 1 \\
1 & 0
\end{array}\right)\right] \\
\operatorname{diar}\left[\left(\begin{array}{ll}
0 & 1 \\
1 & 0
\end{array}\right), \ldots,\left(\begin{array}{ll}
0 & 1 \\
1 & 0
\end{array}\right),\left(\begin{array}{ll}
2 & 1 \\
1 & 2
\end{array}\right)\right]
\end{array} \text { if } p=2 .\right.
$$

$\mathrm{t}|2 T|=p^{a} \cdot u(p \nmid u)$. Then there is an integral matrix $G$ such that $\left.G^{-1}\right]$ is half-integral and $p \nmid\left|2 T\left[G^{-1}\right]\right|$ if and only if the following condins hold:

(i) $a$ is even,

(ii) if $p \neq 2$, then the Hasse invariant is 1 ,

(iii) if $p=2$, then $(-1)^{k} u \equiv 1 \bmod 4$ and the Hasse invariant is $(-1)^{k(k+1) / 2}$ if $(-1)^{k} u \equiv 1 \bmod 8,(-1)^{k(k+1) / 2+1}$ if $(-1)^{k} u \equiv 5 \bmod 8$. re the Hasse invariant $S$ is defined as follows: Taking a regular matrix such that $2 T[H]=\operatorname{diag}\left[d_{1}, \cdots, d_{n}\right]$, we put

$$
S=\prod_{1 \leqq i \leqq n}\left(d_{i}, \prod_{1 \leqq j \leqq i} d_{j}\right),
$$

sere (, ) is the Hilbert symbol of degree 2 on $\boldsymbol{Q}_{p}^{\times}$. $S$ is uniquely deterned by $T$.

Remark 2. Let $K$ be a finite extension field over the $p$-adic rational umber field $\boldsymbol{Q}_{p}, O$ the maximal order of $K$ and $(\delta)$ the different of $K$ over , $(\delta \in K)$. For $x \in K$ we denote by $|x|_{K}$ the normalized valuation of $x$. ir a prime element $\pi$ of $K$ we have $|\pi|_{K}^{-1}=\#(O /(\pi))$. Let $R$ be a symatric matrix in $M_{n}(K)$. Then $R$ is decomposed as $R=C^{-1} D$ such that , $\left.\begin{array}{c}* \\ D\end{array}\right) \in S p_{n}(O)$ and we put $\nu(R)=|\operatorname{det} C|_{K}^{-1}$. This is well-defined. For $\equiv \boldsymbol{Q}_{p}$ we put $e(x)=\exp (2 \pi i$ (the fractional part of $\left.x)\right)$. Let $T$ be a halftegral matrix, that is, $2 T \in M_{n}(O), T={ }^{t} T$ and all diagonal entries of $T$ 'e in $O$. Then we put

$$
b(s, T)=\sum \nu(R)^{-s} e\left(\operatorname{tr}_{K / Q_{p}}\left(\sigma(T R) \delta^{-1}\right)\right),
$$

here $R$ runs over $\left\{R \in M_{n}(K) \mid R={ }^{t} R\right\} \bmod O$. Then all theorems and 
corollaries hold for $b(s, T)$ instead of $b_{p}(s, T)$ with the following minor changes:

(i) $p$ should be $|\pi|_{K}^{-1}$.

(ii) In Theorem $2 G$ runs over $G L_{n}(O) \backslash\left\{G L_{n}(K) \cap M_{n}(O)\right\}$ and $p^{\text {ord }_{p}|G|}$ should be $|\operatorname{det} G|_{K}^{-1}$ and a quadratic form $q$ should be defined over $O /(\pi)$ (also in Corollary).

Conjecture 6.3 for $\lambda=0$, Case SP in [7] where the denominator can be solved therein does not necessarily refer to the reduced denominator.

Remark 3. Let $T$ be a half-integral symmetric binary regular matrix. Denote by $t^{*}$ the discriminant of $\boldsymbol{Q}(\sqrt{-|T|})$ and let $\alpha$ be the integer such that $p^{2 \alpha}|||2 T| / t^{*}$. Then from the explicit formula of $b_{p}(s, T)$ ([1], [3]) follows that $b_{p}(s, p T)-p^{2-s} b_{p}(s, T)$ does not depend on $T$ itself but only on $\alpha,\left(t^{*} / p\right)$ (Kronecker symbol). A weaker assertion holds for the function $\alpha_{1}$ (Case SP) defined in [7] from [3].

\section{REFERENCES}

[1] G. Kaufhold, Dirichletsche Reihe mit Funktionalgleichung in der Theorie der Modulfunktion 2. Grades, Math. Ann., 137 (1959), 454-476.

[ 2 ] Y. Kitaoka, Modular forms of degree $n$ and representation by quadratic forms II, Nagoya Math. J., 87 (1982), 127-146.

[ 3 ] _ A note on local densities of quadratic forms, Nagoya Math. J., 92 (1983), 145-152.

[4] H. Maaß, Die Fourierkoeffizienten der Eisensteinreihen zweiten Grades, Mat.-Fys. Medd. Danske Vid. Selsk., 34 (1964), no. 7.

[5] — Siegel's modular forms and Dirichlet series, Lecture Notes in Mathematics, vol. 216. Berlin, Heidelberg, New York, Springer 1971.

[6] G. Shimura, Confluent hypergeometric functions on tube domains, Math. Ann., 260 (1982), 269-302.

[ 7 ] - On Eisenstein series, to appear in Duke Math. J.

[ 8 ] C. L. Siegel, Über die analytische Theorie der quadratischen Formen, Ann. of Math., 36 (1935), 527-606.

Department of Mathematics

Faculty of Science

Nagoya University

Chikusa-ku, Nagoya 464

Japan 